\title{
Poster Abstract: The CORONA Business in Modern Cities
}

\author{
Piergiorgio Vitello, Andrea Capponi, Pol Klopp, \\ Richard D. Connors, Francesco Viti \\ firstname.lastname@uni.lu \\ University of Luxembourg
}

\begin{abstract}
As a response to the global outbreak of the SARS-COVID-19 pandemic, authorities have enforced a number of measures including social distancing, travel restrictions that lead to the "temporary" closure of activities stemming from public services, schools, industry to local businesses. In this poster we draw the attention to the impact of such measures on urban environments and activities. For this, we use crowdsensed information available from datasets like Google Popular Times and Apple Maps to shed light on the changes undergone during the outbreak and the recovery.
\end{abstract}

\section{CCS CONCEPTS}

- Information systems $\rightarrow$ Crowdsourcing; • Applied computing $\rightarrow$ Health care information systems.

\section{KEYWORDS}

SARS-COVID-19, crowdsensing, data collection, urban computing

\section{ACM Reference Format:}

Piergiorgio Vitello, Andrea Capponi, Pol Klopp, Richard D. Connors, Francesco Viti and Claudio Fiandrino. 2020. Poster Abstract: The CORONA Business in Modern Cities. In The 18th ACM Conference on Embedded Networked Sensor Systems (SenSys '20), November 16-19, 2020, Virtual Event, Japan. ACM, New York, NY, USA, 2 pages. https://doi.org/10.1145/3384419.3430606

\section{INTRODUCTION}

The global outbreak of the Severe Acute Respiratory Syndrome Coronavirus 2 (SARS-COV-2) uncovered the unpreparedness of the vast majority of healthcare systems [1] and lead worldwide public institutions to enforce containing measures such as social distancing, cancellation of public events and closure of daily business, education and recreation activities. As a consequence, business and education systems moved to remote working and teaching, which led to unprecedented changes in the way of consuming Internet services. A recent study [3], demonstrates that the traffic generated by applications such as gaming, messaging, Web conferencing witnessed a significant increase. Reducing and controlling human movement has been of the utmost importance in containing the pandemic spread and to track infections. To this end, many applications have been developed to enforce contact tracing systems (e.g., Corona-Warn-App, Immuni and Radar COVID are examples respectively adopted by Germany [5], Italy and Spain) [4].

Permission to make digital or hard copies of part or all of this work for personal or classroom use is granted without fee provided that copies are not made or distributed for profit or commercial advantage and that copies bear this notice and the full citation on the first page. Copyrights for third-party components of this work must be honored

For all other uses, contact the owner/author(s).

SenSys '20, November 16-19, 2020, Virtual Event, Japan

(c) 2020 Copyright held by the owner/author(s).

ACM ISBN 978-1-4503-7590-0/20/11.

https://doi.org/10.1145/3384419.3430606

\author{
Claudio Fiandrino \\ IMDEA Networks Institute \\ claudio.fiandrino@imdea.org
}

In this work, we present the ongoing research efforts devoted to analyze the implications of confinement policies and self-isolation measures on urban environments. Specifically, we draw the attention to the impact that the changes of our daily habits produced on urban mobility and activities. The utility of such analysis is twofold. On the one hand, we can verify the effectiveness of confinement policies to limit the spread of the virus, i.e., the increase/decrease of the number of infected cases upon relaxation/enforcement of such policies respectively. On the other hand, we can determine which are the changes to urban mobility and daily activities. This information is very important for the concerned stakeholders, e.g., governments bodies, decision-makers and city planners to re-think the existing urban landscape and drive more sustainable city planning. For such analysis, we use crowdsensed data that providers such as Google and Apple make available [2]. Next, we present the dataset (§ 2) and the methodology with early results (§ 3$)$.

\section{THE DATASET}

We present the types of data we use in our analysis, i.e., mobility, activities and publicly available data on SARS-COVID-19 cases.

Google Popular Times (GPT) define the temporal profile of an activity (recreational, groceries, transit and stations and parks) as a vector of normalized per-hour weekly values in the range $[0: 100]$ (0: closing hours, 1 : lowest amount of visits per-hour in a week and 100: the highest). The use of normalized values shows the trend of an activity during a week and inherently the factors that influence such behaviour (e.g., a restaurant that has more success during weekends in touristic areas or at lunchtime in business districts). This hides the degree of success of a single activity (i.e., those that have more customers than others). However, in this work we are not interested in the absolute number of visits, but only the trend over time. Fig. 1 shows the geographical distribution of the considered cities and Table 1 shows the breakdown of the number of vantage point per activity category. Although we started the data collection on March 8th 2020, we have consistent information for all the cities since April 23rd only.

Table 1: A primer on our dataset for a selection of cities with the number of vantage points available per activity

\begin{tabular}{lrrrr}
\hline City & Recreational & Groceries & Transit Stations & PARKs \\
\hline London & - & 38 & 68 & 30 \\
Madrid & 30 & 8 & 15 & - \\
Milan & 21 & 7 & 21 & 1 \\
Stockholm & 13 & 8 & 10 & 1 \\
Copenhagen & - & 50 & 6 & 7 \\
Valencia & 8 & 10 & 9 & 1 \\
Malmö & 7 & 24 & 3 & 5 \\
Luxembourg City & 10 & 54 & 9 & 1 \\
\hline
\end{tabular}

Apple Maps dataset provides information on transportation modalities worldwide in a similar way that GPT does, i.e., data is anonymized and no information about the single users is disclosed. Rather, 


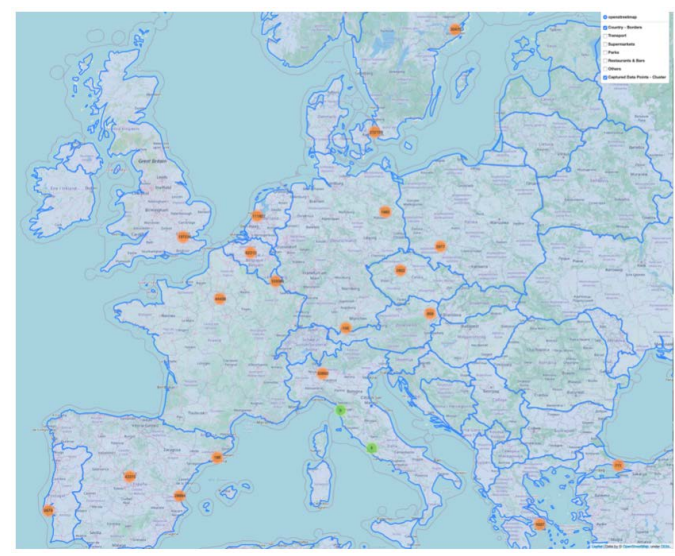

Figure 1: Map of Europe showing the cities with vantage points

the data comes in a form that shows the aggregate requests for destinations for a given transportation mode, e.g., driving, walking, or site, e.g., transit, stations. Further, the data informs about the relative increase or decrease with respect to the values observed pre-SARS-COVID-19 outbreak, i.e., January 13th, 2020. We started the collection of the Apple dataset since February 23rd 2020, when the first confinement policies and self-isolation measures were enforced in Europe.

SARS-COVID-19 Cases The Joint Research Center informs about actual policies enforced and the numbers of infected individuals and fatalities at sub-national level (admin level 1) (https://covidstatistics.jrc.ec.europa.eu/). Since our analysis is at city level, we consider the trend of the corresponding region and extract the cumulative number of infected cases normalized by the total number. Example Fig. 2 shows in a comprehensive graph for Luxembourg City the evolution over time of SARS-COVID-19 cases, lockdown measures, and impact on mobility and cities' activities. Prior to the hard lockdown, groceries have experienced a growth of popularity because people went out to buy goods, with the expectation of imminent restrictions. The confinement measures reduced significantly the mobility (driving category first), and sparked an increase of residential areas activity at the expense of workplaces and recreation categories.

\section{METHODOLOGY AND EARLY RESULTS}

The dataset we are building essentially contains information in form of time series, and our objective is to obtain forecasting models able to predict future number of cases or mobility/activity trend. Currently, Long-short Term Memory (LSTM), a type of recurrent neural network, is the most popular tool to tackle predictive modeling especially with multivariate time series data like in our case. Convolutional LSTM can capture spatio-temporal dependencies. We also consider Transformers as alternative tools, for their property of attention that help capturing long-term dependencies. Such methodology is general enough and applicable not only to Europe.

As a preliminary analysis, we use Gaussian Processes to perform regression over the combined driving category of mobility data and create clusters using the mean as cluster distance metric. Fig. 3 shows the observed results in form of dendrogram. Interestingly,
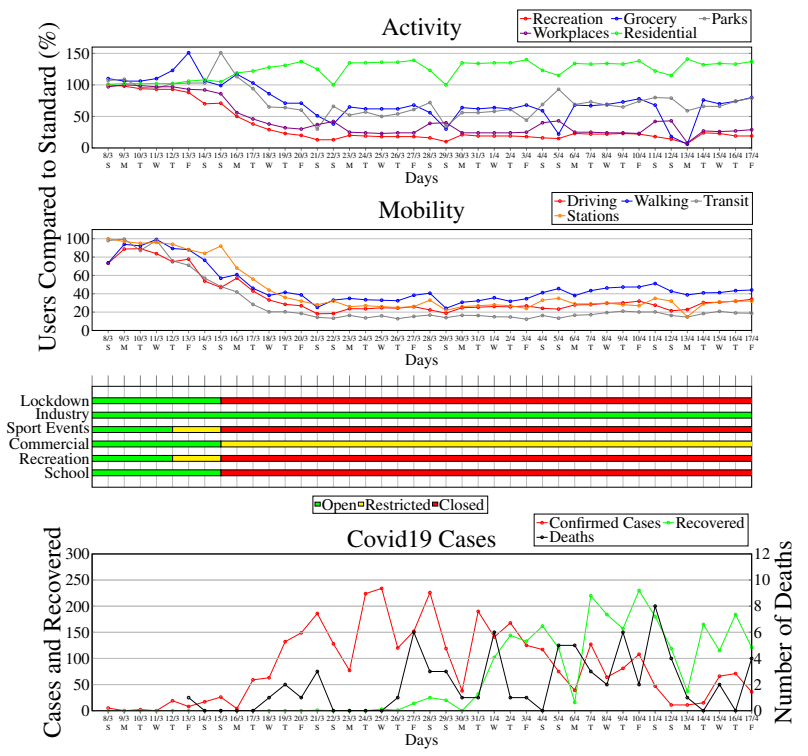

Figure 2: Comprehensive timeline for Luxembourg City (March 8th - April 17th)

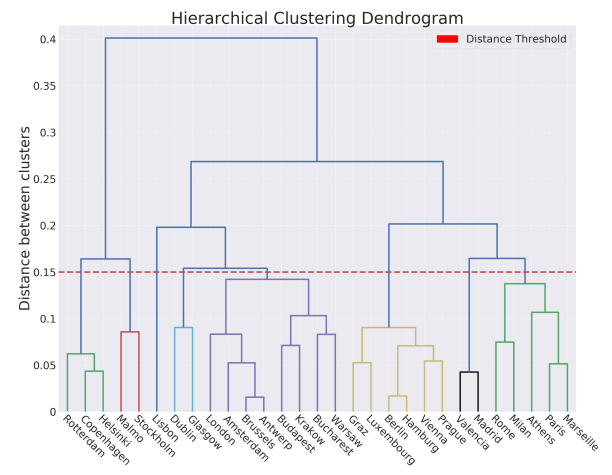

Figure 3: Dendrogram based on driving dataset (February 23th - July 3rd

we observe that many clusters identify geographical countries (e.g., Stockholm and Malmö) or areas (central Europe: Prague, Vienna, Hamburg, Berlin, Luxembourg and Graz). We also observe unexpected results with clusters that do not correspond to a geographical area. For example the cluster that comprises Helsinki, Copenhagen and Rotterdam. This is due to the confinement measures that each municipality has enforced.

\section{REFERENCES}

[1] Maciej F. Boni and et. al. 2020. Evolutionary origins of the SARS-CoV-2 sarbecovirus lineage responsible for the COVID-19 pandemic. Nature Microbiology (28 Jul 2020). https://doi.org/10.1038/s41564-020-0771-4

[2] A. Capponi and et al. 2019. A Survey on Mobile Crowdsensing Systems: Challenges, Solutions and Opportunities. IEEE Communications Surveys Tutorials (May 2019), 1-49. https://doi.org/10.1109/COMST.2019.2914030

[3] Anja Feldmann and et. al. 2020. The Lockdown Effect: Implications of the COVID19 Pandemic on Internet Traffic. In Proc. of ACM IMC. 65-72.

[4] M Kendall and et. al. 2020. Quantifying SARS-CoV-2 transmission suggests epidemic control with digital contact tracing. Science 368, 6491 (2020). https: //doi.org/10.1126/science.abb6936

[5] Jens Helge Reelfs and et. al. 2020. Corona-Warn-App: Tracing the Start of the Official COVID-19 Exposure Notification App for Germany. In Poster in Proc. of ACM SIGCOMM. 1-3. 